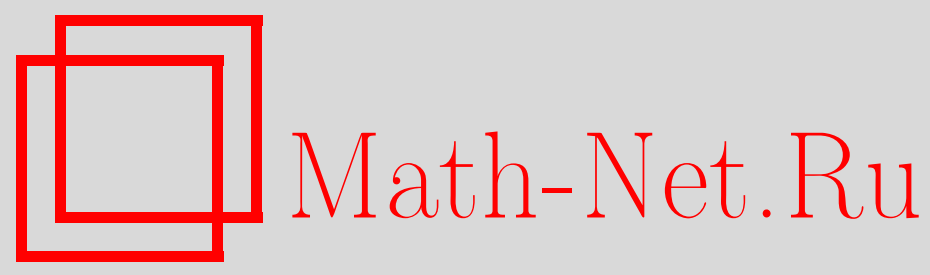

М.-М. Деза, М. И. Штогрин, Метрика постоянной кривизны на полициклах, Матем. заметки, 2005, том 78, выпуск 2, 223-233

DOI: https://doi.org/10.4213/mzm2576

Использование Общероссийского математического портала Math-Net.Ru подразумевает, что вы прочитали и согласны с пользовательским соглашением http://www . mathnet.ru/rus/agreement

Параметры загрузки:

IP : 44.207 .124 .84

26 апреля 2023 г., 13:45:43

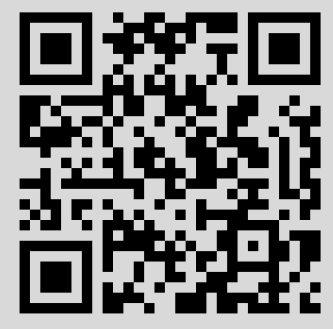




\title{
МЕТРИКА ПОСТОЯННОЙ КРИВИЗНЫ НА ПОЛИЦИКЛАХ
}

\author{
М. Деза, М.И. Штогрин
}

Доказьвается следующая основная теорема теории $(r, q)$-полициклов. Пусть неразделимый плоский граф удовлетворяет следующим двум условиям:

1) каждая внутренняя грань является $r$-угольником, где $r \geqslant 3$;

2 ) каждая внутренняя вершина имеет степень $q$, где $q \geqslant 3$, а каждая граничная вершина имеет степень не больше $q$ и не меньше 2 .

Тогда он удовлетворяет третьему условию:

3) вершины, ребра и внутренние грани образуют клеточный комплекс.

Простые примеры показывают, что условия 1) и 2) независимы даже при выполнении условия 3). Именно они определяют $(r, q)$-полицикл.

Библиографоия: 14 названий.

Мы завершаем серию работ [1]-[10] о так называемых $(r, q)$-полициклах. В этих работах в определениеполицикла включалось три условия. Одно из трех указанных условий оказалось излишним. Два оставшихся условия являются независимьпи. Именно они определяют $(r, q)$-полицикл. Этому основному результату данной работы посвящена теорема ниже.

Теперь приведем наше первоначальное определение $(r, q)$-полицикла и напомним основные свойства исследуемьх нами $(r, q)$-полициклов.

1. Определение и свойства полициклов. Планарный ${ }^{1}$ граф $G$ укладьвается на плоскости так, что никакие два его ребра не пересекаются. Эта укладка - плоская реализация графа $G$ - назьвается плоским әрафом. После присоединения к плоскому графу всех его граней получится так назьваемая плоская карта. Все ограниченные грани плоской карты суть внутренние грани плоского графа. Вершины, не принадлежащие неограниченной грани плоской карты, мы будем назьвать внутренними вериинами плоского графа.

ОПРЕДЕЛЕниЕ. Пусть плоская реализация неразделимого планарного графа $G$ удовлетворяет следующим трем условиям:

1) все внутренние грани суть комбинаторные $r$-угольники, где $r \geqslant 3$;

Работа выполнена при финансовой поддержке Российского фонда фундаментальных исследований, грант № 02-01-00803, программы “Ведушие научные школы”, грант № НШ-2185.2003.1, и программы фундаментальных исследований ОМН РАН "Современные проблемы теоретической математики".

${ }^{1}$ Терминология, относящаяся к теории графов (без петель и кратных ребер), взята из [11]. 
2) все внутренние вершины имеют одну и ту же степень $q$, где $q \geqslant 3$, а всеграничные вершины имеют степень не больше $q$ и не меньше 2 ;

3) вершины, ребра и внутренние грани образуют клеточньй комплекс.

Тогда граф $G$ с присоединенными к нему внутренними гранями (взятыми из данной плоской реализации граф $G$ ) называется $(r, q)$-полиииклом и обозначается через $\Pi(G)$ (см. [1]-[7]).

Следует подчеркнуть, что требование неразделимости графа $G$ в определении $(r, q)$ полицикла $\Pi(G)$ является существенньм. Никакие два $(r, q)$-полицикла с единственной общей вершиной, степень которой не больше $q$, не составляют один объемлющий $(r, q)$-полицикл, так как для их общего графа требование неразделимости нарушено.

Число $r$, фигурирующее в определении $(r, q)$-полицикла П $(G)$, является обхватом графа $G$. Если хоть одна плоская реализация неразделимого планарного графа $G$ удовлетворяет условиям 1)-3), где $r$ - обхват графа, а $q$ - максимальная степень вершины, то мы будем говорить, что граф $G$ допускает $(r, q)$-полиииклическую реализачию.

Имеет место следующая своеобразная теорема единственности полициклической реализации: в общем случае неразделимьй граф $G$ допускает не более чем одну $(r, q)$-полициклическую реализацию (см. [3]-[6]). Лишњ в пяти исключительных случаях, когда граф является реберным остовом платонова тела, число $(r, q)$-полициклических реализаций графа $G$ равно числу граней данного тела. В любом из этих пяти случаев все плоские реализации являются $(r, q)$-полициклическими, причем попарно изоморфньми.

Итак, граф $G$, допускающий $(r, q)$-полициклическую реализацию, $(r, q)$-полициклическая реализация графа $G$ и, наконец, $(r, q)$-полицикл $\Pi(G)$ - это три разных понятия, которые фактически однозначно определяют друг друга. Для любого из трех этих понятий мы вправе использовать единое короткое название полииикл, если из контекста ясно, о каком именно понятии мы говорим.

Следует отметить, что понятие $(r, q)$-полицикла $\Pi(G)$ вводится не только для конечного графа $G$, но и для бесконечного. В случае бесконечного плоского графа мы будем полагать, что в любой конечной области плоскости находится всего лишь конечное число его вершин и ребер. Требование вьполнения этого дополнительного условия является вполне естественньм. Любой $(r, q)$-полищикл П $(G)$ в случае бесконечного графа $G$ представляет собою бесконечную односвязную область, вообще говоря, с краем (см. ниже и [4]-[6]). Любой $(r, q)$-полицикл $\Pi(G)$ в случае конечного графа $G$ представляет собою диск, он всегда с краем; его эйлерова характеристика равна 1.

Для краткости $(r, q)$-полицикл $\Pi(G)$ будем обозначать через $P$. Двумерньй полиэдр, полученньй после замены каждого плоского $r$-угольника из $(r, q)$-полицикла $\Pi(G)$ на двумерньй правильньй $r$-угольник постоянной кривизны с плоскими углами $2 \pi / q$, будем обозначать через $X$. С точки зрения внутренней метрики полиэдр $X$ представляет собою метрическое пространство постоянной кривизны. В самом деле, любая достаточно малая круговая окрестность с центром в произвольной внутренней точке правильного $r$-угольника есть не что иное, как $\varepsilon$-круг постоянной кривизны. Если центр окрестности находится во внутренней точке внутреннего ребраполиэдра $X$, такой $\varepsilon$-круг составлен из двух полукругов. Если центр окрестности совпадает с вершиной, расположенной внутри полиэдра $X$, такой $\varepsilon$-круг состоит из $q$ равных секторов с углами $2 \pi / q$.

Итак, будучи склеенным из правильных $r$-угольников постоянной кривизны с углами $2 \pi / q$ при вершинах, полиэдр $X$, с одной стороны, в точности повторяет клеточную 
структуру полицикла $P$, с другой стороны, представляет собою метрическое пространство постоянной кривизны, а точнее, двумерную поверхность. Как известно (см. соответствующую формулу и теорему, например, в книге $[12$, с. 411]), на любой двумерной поверхности сумма внутренних углов геодезического $m$-угольника выражается числом $(m-2) \pi$, сложенным с полной кривизной $\Omega$ данного $m$-угольника. Это довольно часто встречающийся на практике частньй случай ${ }^{2}$ известной теоремы Гаусса-Бонне.

В качествегеодезического многоугольника возьмем правильный $r$-угольник постоянной кривизны с углами $\varphi_{i}=2 \pi / q$, где $i=1,2, \ldots, r$. Для него по упомянутой теореме имеет место формула

$$
\frac{2(r+q)-r q}{q} \pi=\Omega .
$$

Полиэдр $X$, составленньй из этих $r$-угольников (сходящихся по $q$ в каждой внутренней вершине), комбинаторно изоморфный ему исходный полицикл $P$ и, наконец, соответствующие им параметры $(r, q)$ впредь будем назьвать әллиптическими, параболическими или гиперболическими в зависимости от того, выполнено ли неравенство $2(r+q)>r q$, равенство $^{3} 2(r+q)=r q$ или неравенство $2(r+q)<r q$ соответственно.

Кстати сказать, при любых параметрах $r \geqslant 3, q \geqslant 3$ существует широко применяемое на практике правильное разбиение $\left(r^{q}\right)$. Здесь $\left(r^{q}\right)$ - это стандартное обозначение разбиения сферы $\mathbb{S}^{2}$, евклидовой плоскости $\mathbb{R}^{2}$ или плоскости Лобачевского $\mathbb{H}^{2}$ на правильные $r$-угольники с углами $2 \pi / q$. Правильные разбиения $\left(r^{q}\right)$ в случае евклидовой плоскости или плоскости Лобачевского суть бесконечные $(r, q)$-полициклы. Как оказалось, среди всех $(r, q)$-полициклов они и только они не имеют края. В случае сферы любое правильное разбиение $\left(r^{q}\right)$ также не имеет края. Однако оно не является $(r, q)$-полициклом; после удаления внутренности одной из граней оно превращается в конечньй $(r, q)$-полицикл с краем.

Существование правильного разбиения $\left(r^{q}\right)$ играет основную роль в теории $(r, q)$-полициклов. С ним связано распределение всех полициклов на собственные ${ }^{4}$ и несобственные (гелицены). А именно, в работах [3], [5], [6] была доказана следующая основная лемма: любой $(r, q)$-полицикл клеточно отображается в правильное разбиение $\left(r^{q}\right)$; такое отображение однозначно определяется заданием флага (т.е. инцидентных вершины, ребра, $r$-угольника) и его образа (проекuии) при этом отображении. Рассматриваемое локально гомеоморфное отображение (не) является глобальным гомеоморфизмом в случае (не)собственного полицикла.

Эта принципиальная лемма может быть переформулирована (и доказана) в терминах метрики постоянной кривизны: существует непрерывное локально изометрическое клеточное отображение полиэдра $X$ в правильное разбиение $\left(r^{q}\right)$. Это отображение будем записьвать в виде

$$
f: X \rightarrow\left(r^{q}\right)
$$

\footnotetext{
${ }^{2}$ Точно так же формулируется дискретный аналог этой теоремы для разверток положительной кривизны в книге [13]: избыток суммы углов геодезического $m$-угольника (в сравнении с суммой углов евклидова $m$-угольника) равен его полной кривизне, т.е. $\sum_{i=1}^{m} \varphi_{i}-(m-2) \pi=\Omega$.

3 Это равенство имеет геометрически более понятную запись $(r-2) \pi=2 \pi r / q$ в терминах суммы углов евклидова $r$-угольика с углами $2 \pi / q$. В данном случае $(r, q)=(3,6),(4,4)$ или $(6,3)$.

${ }^{4}$ Любой собственный $(r, q)$-полицикл представляет собою собственную часть разбиения $\left(r^{q}\right)$.
} 
если мы хотим подчеркнуть, что при этом отображении $r$-угольники из полиэдра $X$ переходят в $r$-угольники из разбиения $\left(r^{q}\right)$ в виде

$$
f: \mathscr{B} X \rightarrow \mathscr{B}\left(r^{q}\right),
$$

если хотим подчеркнуть, что треугольники из однократного барицентрического подразделения $\mathscr{B} X$ переходят в треугольники из однократного барицентрического подразделения $\mathscr{B}\left(r^{q}\right)$; или же в виде

$$
f: \mathscr{B}^{2} X \rightarrow \mathscr{B}^{2}\left(r^{q}\right),
$$

если хотим подчеркнуть, что треугольники из двукратного барицентрического подразделения $\mathscr{B}^{2} X$ переходят в треугольники из двукратного барицентрического подразделения $\mathscr{B}^{2}\left(r^{q}\right)$.

С барицентрическими подразделениями можно ознакомиться в $[14]^{5}$. Опишем их специфические особенности в наших частных случаях.

Вершина, середина ребра и центр $r$-угольника в правильном разбиении $\left(r^{q}\right)$ или же в разбиении полиэдра $X$ на правильные $r$-угольники суть вершины барицентрического треугольника в $\mathscr{B}$. Будем говорить, что эти вершины имеют соответственно mun 0,1 и 2. Тип вершины обозначает размерность той клетки, центром которой она является. Каждый барицентрический треугольник имеет ровно по одной вершине каждого типа. В однократном барищентрическом подразделении полиэдра $\left(r^{q}\right)$, которое мы обозначаем через $\mathscr{B}\left(r^{q}\right)$, все вершины являются внутренними. В вершинах типа $2,1,0$ сходятся соответственно $2 r, 4,2 q$ барицентрических треугольников из разбиения $\mathscr{B}\left(r^{q}\right)$. Точно таковы же схождения и во всех внутренних вершинах однократного барицентрического подразделения полиэдра $X$, которое мы обозначаем через $\mathscr{B} X$. Звезда барищентрических треугольников с центром во внутренней вершине разбиения $\mathscr{B} X$ изометрична соответствующей звезде барицентрических треугольников с центром в вершине того же типа из разбиения $\mathscr{B}\left(r^{q}\right)$. Звезда барицентрических треугольников с центром в граничной вершине разбиения $\mathscr{B} X$ изометрична только части соответствующей звезды барицентрических треугольников с центром в вершине того же типа из разбиения $\mathscr{B}\left(r^{q}\right)$.

При переходе от однократного барицентрического подразделения $\mathscr{B}$ к двукратному барицентрическому подразделению $\mathscr{B}^{2}$ любой барищентрический треугольник 012 разбивается на треугольники следующих шести типов:

$$
0.01 .012, \quad 0.02 .012, \quad 1.01 .012, \quad 1.12 .012,2.02 .012,2.12 .012 \text {. }
$$

Здесь запись, например, 0.01.012, обозначает треугольник с вершинами типа 0, 01 (вершина типа 01 - это середина стороны 01) и 012 (вершина типа 012 - это центр треугольника 012). Треугольники всех этих шести типов по-разному расположены относительно $r$-угольника.

В двукратном барицентрическом подразделении имеется всего семь типов вершин: 0, $1,2,01,02,12,012$. В случае разбиения $\mathscr{B}^{2}\left(r^{q}\right)$ все эти вершины (любого типа) являются внутренними. В них сходятся соответственно $4 q, 8,4 r, 4,4,4,6$ барицентрических треугольников. Точно таковы же схождения во внутренних вершинах в случае разбиения $\mathscr{B}^{2} X$. Звезда барищентрических треугольников с центром во внутренней вершине разбиения $\mathscr{B}^{2} X$ изометрична звезде барицентрических треугольников с центром в

${ }^{5}$ Здесь на с. 165 в формулировке основной теоремы топологии поверхностей имеется опечатка: $k(\geqslant 0)$ нужно заменить на $k(>0)$ или на $k(\geqslant 1)$. 
вершине того же типа из разбиения $\mathscr{B}^{2}\left(r^{q}\right)$. Звезда барицентрических треугольников с центром в граничной вершине разбиения $\mathscr{B}^{2} X$ изометрична только части звезды барицентрических треугольников с центром в вершине того же типа из разбиения $\mathscr{B}^{2}\left(r^{q}\right)$.

Однократное барицентрическое подразделение $\mathscr{B}$ и двукратное барицентрическое подразделение $\mathscr{B}^{2}$ являются основными орудиями в дальнейших наших доказательствах.

Отметим три важных момента, способствовавших конструктивному построению отображения $f$, см. (1). Во-первых, вблизи своих внутренних точек $(r, q)$-полицикл П $(G)$ устроен точно так же, как соответствующее ему правильное разбиение $\left(r^{q}\right)$. Во-вторых, любое правильное разбиение $\left(r^{q}\right)$ не имеет края: сфера, евклидова плоскость и плоскость Лобачевского суть полные метрические пространства. В-третьих, $(r, q)$-полицикл $\Pi(G)$ является односвязньм: внутренность любого полицикла представляет собою связную односвязную область. Именно эти обстоятельства позволяют локальное отображение однозначно продолжить до глобального. Мы воспользуемся этим ниже при доказательстве вспомогательной леммы.

2. Основная теорема о полициклах. Теперь сформулируем основной результат данной работы в виде теоремы. В ней утверждается, что фигурируюшие в определении $(r, q)$-полицикла П $(G)$ три условия 1$), 2), 3)$, накладываемые на неразделимьй плоский граф $G$, зависимы.

ТеоремА. Из условий 1) и 2) вытекает условие 3).

Прежде чем приступить к доказательству теоремы, сформулируем одно тривиальное следствие, которое представляется нам очень важным в теории полициклов; ради него данная теорема доказана; его следует воспринимать как упрощенное определение полицикла.

СЛЕДСТВИЕ. Любой $(r, q)$-полицикл $\Pi(G)$ определяется двумя условиями 1), 2), налагаемыми на неразделимый плоский граф $G$.

Итак, в исходном определении полицикла условие 3) оказалось излишним. Простые примеры показывают, что условия 1) и 2) независимы даже при выполнении условия 3). Следовательно, упрощенное определение полицикла в некотором смысле стало окончательным.

ДокАЗАтЕльство теОремы. Теперь приступим к доказательству теоремы ${ }^{6}$. Неразделимьй плоский граф $G$, удовлетворяющий двум условиям 1) и 2), с присоединенными к нему внутренними гранями назовем обобщенны. ${ }^{7}$ полициклом и обозначим через $P_{*}$. Заменив каждый плоский $r$-угольник из $P_{*}$ на правильный двумерньй $r$-угольник постоянной кривизны с углами $2 \pi / q$, мы вместо обобщенного полищикла $P_{*}$ получим полиэдр, снабженный метрикой постоянной кривизны, которьй обозначим через $X_{*}$.

Для него опять вьполнены условия 1) и 2). По условию 1) каждая грань полиэдpa $X_{*}$, как двумерньй комбинаторный $r$-угольник, есть диск. Следовательно, примыкающие к произвольной вершине полиэдра $X_{*}$ разные углы принадлежат разным $r$-уголь-

\footnotetext{
${ }^{6}$ Если рассматриваемый граф̆ $G$ конечен и 3 -связен, то предлагаемая нами теорема вытекает из теоремы Штейница о существовании выпуклого многогранника с реберньм остовом $G$.

7 Это первая попытка обобщить первоначальное понятие полицикла, данное в определении.
} 
никам. Теперь условие 2) мы можем переформулировать так: число $r$-угольников, сходящихся во внутренней вершине полиэдра $X_{*}$, равно $q$, причем все $q$ этих $r$-угольников составляют простой цикл; число $r$-угольников, сходящихся в граничной вершине полиэдра $X_{*}$, равно $j$, где $1 \leqslant j \leqslant q-1$, причем все $j$ этих $r$-угольников составляют простую цепь. Внутреннее ребро полиэдра $X_{*}$ принадлежит двум и только двум $r$-угольникам; граничное ребро принадлежит одному и только одному $r$-угольнику. Наша цель - доказать, что вершины, ребра и грани полиэдра $X_{*}$ образуют клеточный комплекс ${ }^{8}$ (клетки замкнуты). Короче говоря, это полньй аналог триангуляции.

Построим однократное барицентрическое подразделение полиэдра $X_{*}$. Как мы уже условились, обозначаем его через $\mathscr{B} X_{*}$. Покажем, что это триангулящия. В самом деле, во внутренней вершине типа 0 сходится $q$ ребер типа 01 с различными конщами типа 1 , $q$ ребер типа 02 с различными концами типа 2 , а также $2 q$ барицентрических треугольников полиэдра $\mathscr{B} X_{*}$, где $q \geqslant 3$. Все эти $2 q$ треугольников составляют звезду с центром в точке 0. Пересечение любых двух треугольников звезды есть либо ребро, либо вершина. Звезда с центром в граничной вершине типа 0 изометрична части звезды с центром во внутренней вершине типа 0. Ни двуугольники, ни петли к вершинам типа 0 не примыкают, их нет вовсе. То же самое можно сказать о звездах в вершинах типа 1 и 2. Лиш вершина типа 2 всегда является внутренней. Следовательно, барицентрические треугольники полиэдра $\mathscr{B} X_{*}$ составляют триангуляцию.

ОСНовНАЯ ЛЕМмА. Существует непрерывное локально изометрическое клеточное отобрахсение

$$
f_{*}: \mathscr{B} X_{*} \rightarrow \mathscr{B}\left(r^{q}\right)
$$

ДоКАЗАТЕЛЬСТВО. Полиэдры $\mathscr{B} X_{*}$ и $\mathscr{B}\left(r^{q}\right)$ заданы своими триангуляциями. Они состоят из изометричных треугольников. Отображение $f_{*}$ полиэдра $\mathscr{B} X_{*}$ в полиэдр $\mathscr{B}\left(r^{q}\right)$ строится так (см., например, [1]). Берем изометрическое отображение фиксированного треугольника из $\mathscr{B} X_{*}$ на фиксированный треугольник из $\mathscr{B}\left(r^{q}\right)$, сохраняющее типы вершин. Это отображение однозначно продолжается до отображения соседнего (смежного по стороне, если такой имеется) треугольника из $\mathscr{B} X_{*}$ на соседний (смежньй по стороне, такой всегда имеется) треугольник из $\mathscr{B}\left(r^{q}\right)$. Теперь возьмем произвольный треугольник из полиэдра $\mathscr{B} X_{*}$. Соединим его с исходньпм треугольником посредством такой цепочки треугольников, в которой любые два соседние треугольника смежны по стороне. Наличие такой цепочки будет обеспечено даже в случае бесконечного графа в силу указанных выше дополнительных требований. Постепенно перемещаясь вдоль цепочки, мы через конечное число шагов отобразим последний треугольник цепочки на некоторый треугольник из $\mathscr{B}\left(r^{q}\right)$. Покажем, что отображение последнего треугольника цепочки не зависит от выбора той цепочки, посредством которой он соединен с исходным.

Проверим сказанное сначала для так назьваемой элементарной замкнутой цепочки треугольников в разбиении $\mathscr{B} X_{*}$. По определению әлементарная замкнутая иепочка треугольников состоит из:

а) двух треугольников, смежных по внутренней стороне разбиения $\mathscr{B} X_{*}$, с переходом от одного треугольника к другому и обратно;

\footnotetext{
${ }^{8}$ Определение клеточного комплекса см., например, в книге [14, с. 267]. Здесь достаточно напомнить следующее. Нульмерная клетка состоит из одной точки. Граница одномерной клетки представляет собою две точки, а сама клетка - отрезок. Граница двумерной клетки представляет собою окружность, а сама клетка - круг. Пересечение любых двух клеток есть клетка.
} 
b) всех треугольников, сходящихся во внутренней вершине разбиения $\mathscr{B} X_{*}$, с полным обходом треугольников вокруг этой вершины.

Общая вершина треугольников цепочки типа b) может иметь тип 0,1 или 2. Все треугольники цепочки составляют звезду с центром в данной вершине. Эта звезда гомеоморфна диску.

Общая сторона треугольников цепочки типа а) может иметь тип 01, 02 или 12. Два треугольника, примыкающие к стороне любого из этих трех типов, вместе опять составляют диск.

Итак, у полиэдра $\mathscr{B} X_{*}$ имеется всего шесть типов элементарных замкнутых цепочек треугольников. Треугольники элементарной замкнутой цепочки любого из шести типов составляют диск. Ясно, что элементарная замкнутая цепочка из полиэдра $\mathscr{B} X_{*}$ всегда изометрична элементарной замкнутой цепочке того же типа из полиэдра $\mathscr{B}\left(r^{q}\right)$. Более того, изометрическое отображение элементарной цепочки из $\mathscr{B} X_{*}$ на элементарную цепочку из $\mathscr{B}\left(r^{q}\right)$ однозначно определяется по своему ограничению на одном треугольнике. Это означает, что после полного обхода вдоль любой элементарной замкнутой цепочки мы опять окажемся в исходном треугольнике цепочки из полиэдра $\mathscr{B} X_{*}$ вместе с исходным отображением этого треугольника на треугольник из полиэдра $\mathscr{B}\left(r^{q}\right)$.

Теперь, разобравшись с элементарной замкнутой цепочкой, вернемся к произвольной цепочке. Если в каком-либо месте произвольной цепочки мы вставим или выбросим элементарную замкнутую цепочку типа а) или типа b), то при таком преобразовании цепочки новое отображение, соответствующее новой цепочке, совпадет с прежним.

Далее, с помощью преобразований типа а) и типа b) любую цепочку, соединяющую фиксированные два треугольника, можно перевести в любую другую такую цепочку. Это следует из односвязности полиэдра $\mathscr{B} X_{*}$. Поэтому построенное по соединяющей цепочке отображение фактически не зависит от выбора этой цепочки.

Итак, изометрическое отображение фиксированного треугольника полиэдра $\mathscr{B} X_{*}$ на фиксированный треугольник полиэдра $\mathscr{B}\left(r^{q}\right)$, сохраняющее типы вершин, однозначно продолжается до изометрического отображения любого другого его треугольника, т.е. до изометрического отображения всего полиэдра в целом. Лемма доказана.

ЗАмЕчАнИЕ. Звезда треугольников в вершине типа 2 полиэдра $\mathscr{B} X_{*}$ при отображении $(2 *)$ переходит в звезду треугольников в вершине типа 2 полиэдра $\mathscr{B}\left(r^{q}\right)$. Короче говоря, $r$-угольник из полиэдра $X_{*}$ при отображении $f_{*}$ переходит в $r$-угольник из полиэдра $\left(r^{q}\right)$. Это простое заключение играет важную роль при доказательстве теоремы.

Прежде чем приступить к завершению доказательства теоремы, напомним, что разбиение $\left(r^{q}\right)$ обладает следующим замечательным свойством: остов разбиения $\left(r^{q}\right)$, как граф, имеет обхват $r$, т.е. длина минимального реберного цикла в разбиении $\left(r^{q}\right)$ равна $r$ (см. [1]).

В эллиптическом случае это проверяется тривиально. У тетраэдра два смежных ребра всегда принадлежат одному треугольнику. У октаэдра (или икосаэдра) два смежных ребра, принадлежащие одной грани, входят в ее граничньй треугольник, а не принадлежащие одной грани входят в реберньй цикл, длина которого не меньше 4. У куба (додекаэдра) три последовательных ребра, принадлежащие одной грани, входят в ее граничный четырехугольник (пятиугольник), а не принадлежашие одной грани входят в реберный цикл, длина которого не меньше 6.

В параболическом и гиперболическом случае это проверяется так. Возьмем в разбиении $\left(r^{q}\right)$ любой простой реберньй цикл. По теореме Жордана он ограничивает в 
плоскости некоторую конечную область. Эта область содержит по меньшей мере один двумерньй $r$-угольник разбиения $\left(r^{q}\right)$. Выпустим из центра такого $r$-угольника прямолинейные лучи, проходяшие через его вершины. Эти лучи разбивают полньй центральньй угол на $r$ секторов. Каждьй сектор опирается на свою сторону $r$-угольника. Любая линия, соединяющая две точки граничных лучей сектора, которые расположены вне $r$-угольника, является более длинной, чем сторона $r$-угольника. Поэтому число звеньев любого реберного цикла, обхватывающего $r$-угольник, больше $r$, если он не является периметром $r$-угольника. Следовательно, реберньй остов разбиения $\left(r^{q}\right)$ имеет обхват $r$.

В любом стандартном правильном разбиении $\left(r^{q}\right)$ каждьй замкнутьй реберньй цикл длины $r$ ограничивает грань. Через каждое ребро разбиения $\left(r^{q}\right)$ проходит ровно два реберных цикла кратчайшей длины $r$.

В полиэдре $X_{*}$ каждый замкнутьй реберный цикл длины $r$ также ограничивает грань. В общем случае эта грань внутренняя. Лишь в пяти исключительных частных случаях эта грань внешняя. $\mathrm{K}$ каждому внутреннему ребру полиэдра $X_{*}$ примыкают две его внутренние грани. $\mathrm{K}$ каждому граничному ребру примыкает одна внутренняя грань.

Теперь исследуем вид связной компоненты пересечения двух $r$-угольников из полиэдра $X_{*}$. Пусть пересечение двух $r$-угольников из полиэдра $X_{*}$ не пусто. Покажем, от противного, что связная компонента пересечения есть ребро или вершина. Пусть связная компонента пересечения двух $r$-угольников из $X_{*}$ содержит не менее двух ребер. Рассмотрим общую вершину двух ребер, принадлежащих одной компонентепересечения. $\mathrm{K}$ данной вершине примыкают два последовательных ребра как одного $r$-угольника, так и другого. К любому из двух этих ребер примыкают оба двумерных $r$-угольника. Поэтому данная вершина является внутренней точкой полиэдра $X_{*}$. Степень данной вершины равна 2. Получено противоречие с условием 2 ) теоремы, по которому $q \geqslant 3$. Следовательно, связная компонента непустого пересечения двух $r$-угольников может быть только одно ребро или одна вершина.

Далее, исследуем число связных компонент пересечения любых двух $r$-угольников полиэдра $X_{*}$. Покажем, от противного, что пересечение имеет лиш одну связную компоненту. Пусть пересечение двух $r$-угольников имеет не менее двух связных компонент. Возьмем две общие вершины двух $r$-угольников, принадлежащие разным компонентам их пересечения. Эти две вершины разбивают периметр одного $r$-угольника на две цепи $x$ и $y$, а периметр другого $r$-угольника на две цепи $u$ и $v$. Не теряя общности, можно считать, что длина (число звеньев) цепи $x$ не больше длины цепи $u$ (иначе обозначения поменяем местами). Тогда цикл $x v$ не длиннее цикла $u v$. Так как длина цикла $u v$ равна $r$, то длина цикла $x v$ не больше $r$. Покажем, что она равна $r$. Для этой цели воспользуемся отображением $f_{*}$, построенным в лемме. Произвольный реберньй цикл $\gamma$ из полиэдра $X_{*}$ при отображении $f_{*}$ переходит в реберный цикл $f_{*}(\gamma)$ из разбиения $\left(r^{q}\right)$, причем той же длины, что и $\gamma$. А так как длина $f_{*}(\gamma)$ не может быть короче, чем $r$, то и сам цикл $\gamma$ не короче $r$. В частности, наш цикл $x v$ длины не более $r$ при отображении $f_{*}$ переходит в цикл $f_{*}(x v)$ длины не более $r$. А так как реберный остов разбиения $\left(r^{q}\right)$ имеет обхват $r$, то длина цикла $f_{*}(x v)$ не меньше $r$. Значит, длина цикла $f_{*}(x v)$ равна $r$. Поэтому длина цикла $x v$ также должна быть равна $r$. Цикл $x v$ должен ограничивать грань, внутреннюю или внешнюю. Последнее возможно лишш в случае платонова тела без внутренности одной из его граней. Но в таком случае любые две внутренние 
грани имеют связное пересечение, а это не соответствует рассматриваемому здесь случаю. Остается единственная возможность - цикл $x v$ ограничивает внутреннюю грань. В этом случае цеп $x$ есть ребро, представляющее собою связную компоненту пересечения двух граней с краями $x y$ и $x v$. Точно так же цепь $v$ есть ребро, представляющее собою связную компоненту пересечения двух граней с краями $x v$ и $u v$. Два ребра $x$ и $v$ составляют двуугольник, т.е. обхват реберного остова полиэдра $X_{*}$ равен 2 . А это противоречит условию 1 ) теоремы, согласно которому $r \geqslant 3$. Следовательно, связная компонента пересечения двух $r$-угольников может быть только одна.

Итак, непустое пересечение двух $r$-угольников из полиэдра $X_{*}$ всегда связно и представляет собою либо одно ребро, либо одну вершину.

Так как граф $G$ не имеет петель и кратных ребер, то пересечение двух разных его ребер может состоять только из одной вершины или быть пустым.

Для завершения доказательства теоремы осталось исследовать пересечение $r$-угольника и ребра. Достаточно рассмотреть лишш тот случай, когда ребро имеет с $r$-угольником две общие вершины, но само ему не принадлежит. Ясно, что данное ребро принадлежит какому-то другому $r$-угольнику полиэдра $X_{*}$ (причем одному, если оно является граничным для $X_{*}$, или двум, если оно является внутренним для $X_{*}$ ). Значит, получаем два $r$-угольника с двумя обшими вершинами. В силу сказанного вьше они имеют общее ребро, соединяющее две эти вершины. Поэтому данные две вершины соединяют два разных ребра. Опять мы имеем обхват 2. Это противоречит условию 1) теоремы, согласно которому $r \geqslant 3$. Следовательно, если ребро имеет с $r$-угольником две общие вершины, то это ребро само обязано принадлежать этому $r$-угольнику.

Итак, рассматриваемое нами разбиение полиэдра $X_{*}$ на правильные $r$-угольники, изоморфное обобщенному полициклу $P_{*}$, представляет собою клеточньй комплекс. Доказательство теоремы завершено.

Так как условия 1$), 2$ ) влекут условие 3$)$, то знак * в отображении $(2 *)$ можно опустить, оно совпадает с отображением (2). Обобщенный полицикл $P_{*}$ совпадает с нашим первоначальным полициклом $P$.

3. О попытке обобщения полицик лов. Первая попытка обобщения понятия полицикла, предпринятая в предыдущем пункте, не привела нас к расширению этого понятия. Сейчас мы предпримем еще одну попытку. И прежде всего попытаемся расширить понятие грани.

В случае связного плоского графа любая его внутренняя грань является односвязной областью. Ранее мы полагали, что замыкание этой области опять остается односвязным. Теперь будем предполагать, что замыкание рассматриваемой односвязной области не является односвязным. Введем на плоскости определенную ориентацию. Следуя этой ориентации, будем обходить внутреннюю грань плоского графа вдоль ее границы. Ребро, в окрестности которого внутренняя грань расположена по разные стороны от ребра, будет проходиться дважды: сначала в одном, потом в другом направлении. После полного обхода вокруг внутренней грани вдоль ее границы мы получим замкнутьй реберный путь. Число ребер этого пути вновь будем обозначать через $r$.

Как оказалось, условие 1) в доказанной нами теореме можно заменить на более слабое условие:

$\left.1^{\prime}\right)$ каждая внутренняя грань плоского графа $G$ ограничена замкнутым реберным путем длины $r$, где $r \geqslant 3$. 
В условии $1^{\prime}$ ) граница внутренней грани уже не обязана быть простым реберным циклом.

Пусть неразделимьй плоский граф $G$ удовлетворяет двум условиям $\left.1^{\prime}\right)$ и 2 ). Тогда имеет место следующее

ПРЕДЛОЖЕНИЕ. Из условий $\left.1^{\prime}\right), 2$ ) следуют условия 1), 2).

ДокАЗАтЕльство. Неразделимьй плоский граф $G$, удовлетворяющий условиям $\left.1^{\prime}\right), 2$ ), с присоединенными к нему внутренними гранями опять назовем обобщенны. ${ }^{9}$ полициклом. Обозначим его через $P_{* *}$. Заменив каждьй плоский $r$-угольник из $P_{* *}$ на двумерньй правильньй $r$-угольник постоянной кривизны с углами $2 \pi / q$, мы вместо обобщенного полицикла $P_{* *}$ получим полиэдр $X_{* *}$. Двумерный $r$-угольник в полиэдpe $X_{* *}$ не обязан быть диском, так как не исключены отождествления некоторых точек его границы. Более того, однократное барищентрическое подразделение $\mathscr{B} X_{* *}$ не обязано быть триангуляцией. Однако двукратное подразделение $\mathscr{B}^{2} X_{* *}$, как показала простая проверка, является триангуляцией.

Фиксируем барицентрический треугольник двукратного барицентрического подразделения $\mathscr{B}^{2} X_{* *}$. Сохраняя типы вершин треугольника, изометрически отобразим его на треугольник того же типа из разбиения $\mathscr{B}^{2}\left(r^{q}\right)$. Это отображение продолжим до непрерьвного локально изометрического клеточного отображения

$$
f_{* *}: \mathscr{B}^{2} X_{* *} \rightarrow \mathscr{B}^{2}\left(r^{q}\right) .
$$

Существование отображения $\left(3_{* *}\right)$ доказывается точно так же, как и в случае отображения $(2 *)$. При отображении $f_{* *}$ реберный остов полиэдра $X_{* *}$ переходит в реберньй остов разбиения $\left(r^{q}\right)$. Если бы двумерньй $r$-угольник полиэдра $X_{* *}$, как грань, имел внешнее самокасание, то часть его границы, начинающаяся в точке самокасания и заканчивающаяся в ней же, составляла бы замкнутый реберньй цикл $\gamma$, длина которого меньше $r$. Тогда его образ $f_{* *}(\gamma)$ также был бы короче $r$. Но это невозможно, так как реберный остов разбиения $\left(r^{q}\right)$ имеет обхват $r$. Следовательно, никакой двумерньй $r$-угольник в полиэдре $X_{* *}$ не имеет самокасания. Предложение доказано.

Итак, любой двумерньй $r$-угольник рассматриваемого полиэдра $X_{* *}$ является диском. Реберньй путь в условии $1^{\prime}$ ) на самом деле представляет собою реберньй цикл. Знак ** является излишним. Отображение $\left(3_{* *}\right)$ совпадает с $(3)$. Обобщенньй полицикл $P_{* *}$ опять совпадает с первоначальным полициклом $P$. В силу доказанного предложения условия нашей теоремы могут быть ослаблены лишь формально.

ПРИМЕчАниЕ. Двумерньй выпукльй многоугольник постоянной кривизны представляет собою диск. После отождествления некоторых точек гранищы этого многоугольника, не нарушающем постоянства кривизны, он не только перестанет быть диском, но и не допустит расширения до односвязного метрического пространства постоянной кривизны ${ }^{10}$. Именно это обстоятельство является истинной причиной того, что формальное ослабление условий теоремы не дало никакого реального расширения понятия исследуемого нами объекта - полицикла.

\footnotetext{
${ }^{9}$ Это вторая попттка обобщить первоначальное понятие полицик ла, данное в определении.

${ }^{10}$ Напомним, что для упрощения исследований полициклов мы произвольно взятый $(r, q)$-полицикл $P$ заменяем на комбинаторно изоморфный ему двумерный полиэдр $X$, который представляет собою односвязное метрическое пространство постоянной кривизны.
} 


\section{СПИСОК ЦИТИРОВАННОЙ ЛИТЕРАТУРЫ}

[1] Деза М., Штогрин М. И. Примитивные полициклы и гелицены // УМН. 1999. Т. 54. №6. С. 159-160.

[2] Штогрин М. И. Примитивные полициклы: критерий // УМН. 1999. Т. 54. №6. С. 177-178.

[3] Деза М., Штогрин М. И. Бесконечные примитивные полициклы // УМН. 2000. Т. 55. № 1. С. $179-180$.

[4] Штогрин М.И. Непримитивные полициклы и гелицены // УМН. 2000. Т. 55. №2. C. $159-160$.

[5] Деза М., Штогрин М.И. Полициклы: симметрия и вложимость // УМН. 2000. Т. 55. №6. C. $129-130$.

[6] Deza M., Shtogrin M. I. Clusters of cycles // J. Geom. Phys. 2001. V. 40.№ 3, 4. P. 302-319.

[7] Деза М., Штогрин М. И. Экстремальные и нерасширяемые полициклы // Тр. МИАН. 2002. T. 239. C. $127-145$.

[8] Деза М., Штогрин М. И. Критерий вложимости $(r, q)$-полициклов // УМН. 2002. Т. 57. № 3. C. 149-150.

[9] Деза М., Штогрин М. И. Вложение химических графов в гиперкубы // Матем. заметки. 2000. Т. 68 . № 3. С. $339-352$.

[10] Деза М., Штогрин М.И. Мозаики и их изометрические вложения // Изв. РАН. Сер. матем. 2002. Т. 66. № 3. С. 3-22.

[11] Харари $\Phi$. Теория графов. М.: Мир, 1973.

[12] Каган В.Ф. Основы теории поверхностей. Ч. І. М.-Л.: Гостехиздат, 1947.

[13] Александров А. Д. Выпуклые многогранники. М.-Л.: Гостехиздат, 1950.

[14] Зейферт Г., Трельфаль В. Топология. М.-Л.: ОНТИ НКТП СССР, 1938.

(М. Деза) École Normale Superiour, Paris; Institute of Statistical Mathematics, Tokyo 